\title{
RELACIÓN ENTRE LA DINAMOMETRÍA DE MANO Y LA CIRCUNFERENCIA DE PANTORRILLA CON EL ÍNDICE DE MASA CORPORAL EN ANCIANOS AUTOVALENTES
}

\author{
RELATIONSHIP BETWEEN BODY MASS \\ INDEX WITH CALF CIRCUNFERENCE AND \\ DYNAMOMETRY IN SELF-MAINTAINING ELDERLY
}

\author{
José Luis Pino V. (1), María Angélica Mardones H. (2), Claudio Díaz H. (1) \\ (1) Escuela de Nutrición y Dietética, Universidad del Mar, Sede Centro Sur, Talca, Chile. \\ (2) Departamento de Nutrición y Salud Pública, Facultad de Ciencias de la Salud y los Alimentos, \\ Universidad del Bio-Bio, Chillán, Chile.
}

\begin{abstract}
Background: The control of the birthrate and the decrease of the mortality have unleashed a demographic aging. Nutritional status indicators are useless and in some cases it is not possible to determine the exact height or weight, making these indicators useless. Aim: To correlate the BMI with calf perimeter and muscle strength (dynamometry dominant and non-dominant). Methods: We studied 60 adults over 60 years old, with self-maintenance functional capacity. The subjects belonged to the "Circle of Retired Chilean Police" of Chillan city in Chile. The anthropometric measurements and dynamometry were obtained according to WHO specifications. We used correlation coefficients and ANOVA to determine the association between variables. Results: There was a weak negative relationship between dynamometry with BMI, $r=-0.19$ dynamometry dominant $(p=0.332)$ and $r=-0.11$ dynamometry non-dominant $(p=0.806)$, but a positive correlation was found between the calf perimeter and BMI, $r=0,64(p<0.001)$. Conclusions: The data show the utility of calf perimeter as an indicator of nutritional status in the study of subjects, which was not observed with dynamometry.

Key words: Nutritional status, Body Mass Index (BMI), muscle strength (dynamometry), calf perimeter, elderly.
\end{abstract}

Este trabajo fue recibido el 13 de Septiembre de 2010 y aceptado para ser publicado el 25 de Enero de 2011.

\section{INTRODUCCIÓN}

Los seres humanos evolucionan y cambian con el pasar de los años. Así, Macionis y Plummer, plantean que el proceso de envejecimiento de la población es producto de la modernización, siendo el incremento en las expectativas de vida un logro alcanzado en todas las sociedades industriales de mayor y menor grado (1). En este proceso de declive fisiológico se desarrollan enfermedades degenerativas que limitan las capacidades funcionales, entre ellas: diabetes mellitus, hipertensión arterial, dislipidemias, alzhéimer, demencia senil, artritis, artrosis, entre otras.

El control de la natalidad y el descenso de la mortalidad, producidos por la evolución del hombre, han desencadenado un envejecimiento demográfico y como consecuencia Chile posee una importante tasa de población envejecida, siendo la tendencia para el año 2025 de más del 16\% la población adulto mayor (2).

En este contexto, la alimentación y nutrición de este grupo etáreo es de vital importancia, ya que se ha demostrado una estrecha relación entre la ingesta de alimentos y las condiciones de vida, cambios digestivos, estado psicológico, desarrollo de ECNT y expectativas de vida (3-5). Por otro lado se ha descrito que recuperar nutricionalmente a un anciano deteriorado es una tarea muy difícil (6). De ahí deriva la importancia de evaluar el estado nutricional en los adultos mayores. Según Chumlea W y Sun S, es esencial conocer la composición corporal en el anciano, y elaborar un método no invasivo, confiable y de bajo costo, para analizar los cambios 
nutricionales que aparecen con la edad (7).

El estado nutricional de los ancianos está determinado por el balance energético, y este a su vez por requerimientos secundarios (estrés metabólico), actividad física, aislamiento social, medicamentos, entre otros. Actualmente en Chile, existe un elevado porcentaje de malnutrición por exceso en adultos mayores, más de la mitad de ellos presenta un exceso de peso, $30.1 \%$ sobrepeso y $23.4 \%$ Obesidad (8).

Para determinar el estado nutricional de los adultos mayores comúnmente se utiliza el peso y la talla, para con ellos calcular el índice de masa corporal (IMC), comparándolo posteriormente con las referencias propuestas por el Ministerio de Salud de Chile (9), En ciertas oportunidades la medición de estos parámetros antropométricos se dificulta por: curvatura de columna, postración u otras afecciones físicas. Para dar solución a esta problemática algunos autores han relacionado otros parámetros directamente con el IMC, entre ellos el perímetro de pantorrilla y la dinamometría de mano, obteniendo buenos resultados (10-13).

Dado que actualmente nuestra población presenta una expectativa de vida más larga, pero con alto riesgo de desarrollar dificultades fisiopatológicas, surge la preocupación por la búsqueda de un indicador de estado nutricional válido, exacto y de una alta confiabilidad, que pueda ser utilizado en estas personas.

Por lo anterior, nuestro objetivo es relacionar IMC con el perímetro de pantorrilla y la fuerza muscular (medido por dinamometría de mano dominante y nodominante) en ancianos autovalentes.

\section{SUJETOS Y MÉTODO}

Se realizó un estudio de enfoque metodológico cuantitativo, correlacional, transversal, en adultos mayores de 60 años autovalentes. La investigación se realizó en adultos de ambos sexos, pertenecientes al círculo de Carabineros de Chile en retiro, de la ciudad de Chillán.

Se recurrió al muestreo no probabilístico del tipo casual o incidental seleccionando a 60 personas, 22 mujeres y 38 varones, los cuales representaron el $12.9 \%$ del Universo.

El peso, talla, perímetro de pantorrilla, y dinamometría de mano fueron medidos, registrados, recopilados y analizados por los investigadores, utilizando las técnicas establecidas por la Organización Mundial de la Salud $(14,15)$. Se utilizó una balanza SECA 884 digital con precisión de $0.1 \mathrm{~kg}$. con una carga máxima de 140 kilos, un altímetro SECA modelo 240 de $210 \mathrm{~cm}$, con precisión de $1 \mathrm{~mm}$, una cinta métrica Rollfix de $150 \mathrm{~cm}$., con una precisión de $1 \mathrm{~mm}$, angosta, flexible e inextensible y un dinamómetro de mano Baseline, graduado en puntos y kilogramos, cuya tara máxima es de $80 \mathrm{Kg}$ o 120 puntos, con una precisión de $2 \mathrm{~kg}$.

Para disminuir la variabilidad intraindividual y sesgos, los datos fueron recolectados en las mismas condiciones de recinto, materiales, horario y métodos.

Para evaluar el estado nutricional se calculó y analizó el índice de masa corporal (IMC) (16), considerando normal un valor entre 23.1 y $27.9 \mathrm{~kg} / \mathrm{Mt}^{2}$ (17). Se elaboró una base de datos con el fin de almacenar ordenada y lógicamente la información obtenida de la muestra en el programa Microsoft Excel 2003.

El análisis descriptivo, se realizó a través de estadígrafos de tendencia central, medidas de dispersión y posición. El análisis de correlación de variables, se realizó utilizando la prueba de coeficiente de correlación ( $\mathrm{r}$ y $\mathrm{R}^{2}$ ) y ANOVA; para lo cual se utilizó el programa, STATA 8.0 SE. El nivel de significación usado para realizar las inferencias fue de 0,05 .

\section{RESULTADOS}

Se evaluó el estado nutricional (EN) de 60 adultos mayores de 60 años de ambos sexos. De ellos 38 (63.3\%) pertenecieron al género masculino. El peso de los hombres fue significativamente mayor al de las mujeres, $(79,75 \pm 13,11 \mathrm{Kg}$. vs. $70,75 \pm 8,69 \mathrm{Kg}$.) p<0.05, los hombres pesaban $9 \mathrm{Kg}$ más que las mujeres. (tabla 1 )

La talla de los hombres $(1,67 \pm 0.06 \mathrm{mt})$ fue mayor a la de las mujeres $(1,51 \pm 0.05 \mathrm{mt})$ en aproximadamente $16 \mathrm{~cm}(\mathrm{p}<0.001)$.

Respecto al perímetro de pantorrilla, no se encontró una diferencia estadísticamente significativa entre hombres $(37,03 \pm 3,18 \mathrm{~cm})$ y mujeres $(35,89 \pm 2,33 \mathrm{~cm})$ $(\mathrm{p}=0.1476)$. La variabilidad intra individual fue de $8.1 \%$, siendo menor en las mujeres $6.3 \%$.

La dinamometría de mano, dominante y no dominante, fueron significativamente mayores en hombres respecto a las mujeres $(\mathrm{p}<0.0001)$, revelando una diferencia de $15.78 \mathrm{Kg}$ para dinamometría no dominante y 14.98 Kg para dinamometría dominante.

El índice de masa corporal fue mayor en las mujeres $(\mathrm{p}<0,05)$, con una diferencia de 2.4 puntos en el IMC. Los varones presentaron un mayor porcentaje de EN normal que las mujeres, $16(42.1 \%)$ vs $5(22.7 \%)$. En cuanto a malnutrición por exceso, los hombres presentaron mayor sobrepeso 13 (34.2\%) vs 4 (18.2\%) que las mujeres, y una menor proporción de obesidad 7 (18.4\%) vs $12(54.5 \%)$.

Al relacionar el perímetro de pantorrilla con el IMC, se encontró que aproximadamente el $41 \%$ de la variabilidad de IMC estuvo asociada a la variación de la circunferencia de pantorrilla $\left(\mathrm{R}^{2} 0.411 \mathrm{p}<0.001\right)$ (figura $1)$, y con una correlación positiva mediana $(r=0.64)$ 
entre las dos variables, es decir, a mayor IMC mayor perímetro de pantorrilla. Según genero se encontró que, el perímetro de pantorrilla se relaciona en un $37 \%$, con el IMC en mujeres, con una correlación positiva mediana ( $\mathrm{r}=0.61$ ); en tanto que los varones presentaron una asociación de $62 \%$ con una correlación positiva mediana $(\mathrm{r}=0.78)$. Por lo tanto, la relación de perímetro de pantorrilla con IMC tiene mayor efectividad en hombres, según este estudio.

Se detectó una relación muy leve entre la dinamometría de mano no dominante y el IMC, asociándose sólo en $1.23 \%(\mathrm{R} 2=0.0123 \mathrm{p}=0.806)$ con una correlación negativa débil ( $\mathrm{r}=-0.11)$, por lo que la línea de tendencia es casi paralela al eje $\mathrm{X}$; vale decir, un sujeto que tenga un IMC de $24 \mathrm{Kg} / \mathrm{mt}^{2}$ tiene aproximadamente la misma fuerza de empuñamiento, que un sujeto con un IMC de $33 \mathrm{Kg} / \mathrm{mt}^{2}$ (figura 1).

En la dinamometría de mano dominante, también existió una relación muy débil $(\mathrm{R} 2=0.0386 \mathrm{p}=0.332)$ de $3.8 \%$ con una correlación negativa débil $(\mathrm{r}=-0.19)$ graficando una línea de tendencia descendente y casi paralela (aprox. $10^{\circ}$ ) al eje X.

Analizando la dinamometría de mano no dominante con la edad, para observar si existen diferencias de fuerza a medida que aumentan los años, podemos decir que la variación de edad se relacionó sólo en $0.59 \%$ con la variación de la dinamometría de mano no dominante, con una correlación negativa débil $(\mathrm{r}=-0.076 \mathrm{p}=0.586)$, no encontrándose relación alguna.

Esto indicaría que una persona de 60 años tiene

\section{TABLA 1}

\section{Características de adultos mayores del Círculo de Carabineros de Chile en Retiro Casos $(\mathbf{N}=60)$}

\begin{tabular}{|c|c|c|c|}
\hline \multirow[b]{2}{*}{ Variables } & \multicolumn{3}{|c|}{ Grupo estudio } \\
\hline & $\begin{array}{c}\text { Hombres } \\
\mathrm{X} \pm \mathrm{D} . \mathrm{S} \\
\mathrm{n}=\mathbf{3 8}\end{array}$ & $\begin{array}{c}\text { Mujeres } \\
X \pm D . S \\
n=22\end{array}$ & valor de $p$ \\
\hline Edad (años) & $69,36 \pm 7,83$ & $66,73 \pm 6,47$ & $0,1863^{\mathrm{a}}$ \\
\hline Peso (Kg) & $79,75 \pm 13,11$ & $70,75 \pm 8,69$ & $0,005^{\mathrm{b}}$ \\
\hline Talla (Mt) & $1,67 \pm 0.06$ & $1,51 \pm 0.05$ & $0,0000^{\mathrm{a}}$ \\
\hline Dinamometría Nd $(\mathrm{Kg})$ & $36,87 \pm 7,48$ & $21,09 \pm 4,71$ & $0,0000^{\mathrm{b}}$ \\
\hline Dinamometría D (Kg) & $38,34 \pm 6,22$ & $23,36 \pm 4,15$ & $0,0000^{\mathrm{b}}$ \\
\hline P. Pantorrilla (cm) & $37,03 \pm 3,18$ & $35,89 \pm 2,33$ & $0,1476^{\mathrm{a}}$ \\
\hline IMC (Kg./mt2) & $28,68 \pm 4,29$ & $31,08 \pm 4,32$ & $0,0414^{\mathrm{a}}$ \\
\hline
\end{tabular}

TABLA 2

\section{Análisis de regresión lineal simple Variable dependiente IMC}

Variables

Perímetro de pantorrilla

Dinamometría de mano no dominante

Dinamometría de mano dominante $\mathbf{r}$

0.64

$-0.11$

$-0.19$ $\alpha$ 24.062 38.555 44.839

\section{$\boldsymbol{\beta}$}

0.4245

0.2528

0.4056 p-value

$<0.001$

0.806 
aproximadamente la misma fuerza de empuñamiento que una persona de 70 años, en este grupo estudiado.

No se encontró relación entre dinamometría y edad, según sexo, arrojando un $\mathrm{r}=-0.29$ para las mujeres y un $r=-0.33$ para los varones.

Relacionando la dinamometría de mano no dominante con el estado nutricional, se observó que las personas mayores de 60 años con un EN normal y sobrepeso, ejercen una mayor fuerza de empuñamiento que los sujetos enflaquecidos y obesos ( $\mathrm{p}=0.258$ ) (figura 2 ).

\section{DISCUSIÓN Y CONCLUSIÓN}

Los últimos datos informados por el Ministerio de Salud de Chile, indican que más de la mitad de los adultos mayores tiene algún grado de malnutrición por exceso (8), información que es reafirmada por esta investigación y otras tanto nacionales (18) como internacionales (19) señalando la tendencia regional y mundial (20-23). Esta tendencia preocupa enormemente, ya que los adultos mayores constituyen un grupo en crecimiento demográfico y de alto riesgo de enfermedades crónicas

\section{FIGURA 1}

Correlación de perímetro de pantorrilla y dinamometría con el IMC de adultos mayores pertenecientes al Círculo de Carabineros $(\mathbf{R})$ de Chillán. $(n=60)$.

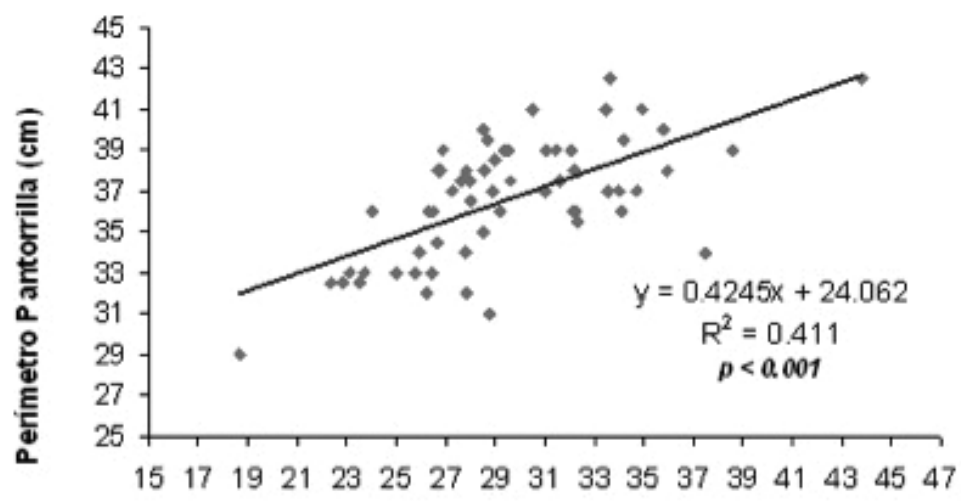

IMC ( $\mathrm{kg} / \mathrm{mt} 2)$

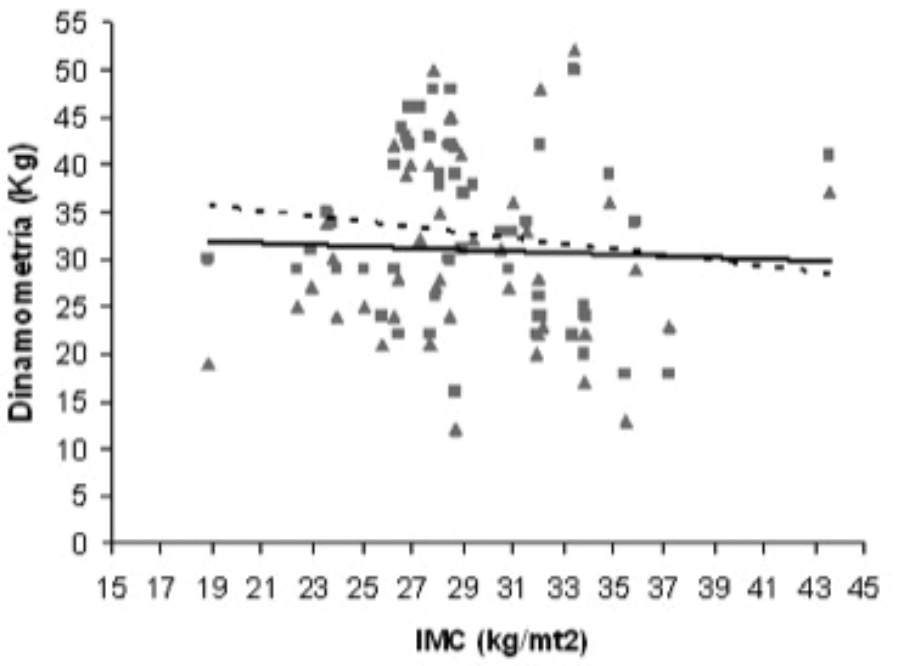

4. Dinam ND $p=0.806$

- Dinam D $\rho=0.332$

... Lineal (Dinam. D)

- Lineal (Dinam. ND) 
no transmisibles (24).

Nuestro estudio evidenció una relación significativa $(\mathrm{p}<0,001)$ entre el perímetro de pantorrilla y el IMC, al igual que múltiples estudios que señalan la utilidad de esta medida como un indicador indirecto de estado nutricional $(25,26)$ y de la composición corporal $(12)$. También se ha encontrado asociación de este parámetro con la albúmina sérica (27) y la mortalidad de adultos mayores (28).

En nuestra investigación la dinamometría de mano no dominante para varones fue de $36.87 \pm 7.48$ y para mujeres de $21.09 \pm 4.71$. Comparado con los resultados obtenidos por Hirsch (10), los varones no presentaron diferencias significativas ( $\mathrm{p}=0.1139)$, pero si las mujeres $(\mathrm{p}<0.05)$, con una diferencia de casi 14 kilos de fuerza de empuñamiento, diferencia quizás establecida debida a la población estudiada.

La dinamometría es una medida que está tomando una importancia relevante, utilizándose tanto en adulos en adultos como en ancianos, (29-31) ya que posee múltiples aplicaciones (32). Se ha encontrado asociación de ella con la masa magra y la funcionalidad del adulto mayor $(33,34)$; por otro lado se ha asociado a complicaciones postoperatorias $(35,36)$, fuerza en cadera y rodilla, mejor equilibrio en ancianos (37), como predictor de riesgo de malnutrición por déficit (38), entre otros.
En este estudio no se encontró una relación significativa entre la dinamometría de mano y el IMC, lo que no descarta su uso como predictor de morbimortalidad $(39,40)$ y/o declinación funcional $(41)$, tal como se describió anteriormente.

Al comparar los resultados de fuerza de empuñamiento obtenidos en adultos mayores de 60 años autovalentes con adultos institucionalizados de la misma edad (42) se observó una diferencia de aproximadamente 10.9 $\mathrm{kg}$ a favor de los autovalentes, diferencia establecida quizás al menor depósito energético-proteico de los ancianos institucionalizados (43).

Podemos concluir que el perímetro de pantorrilla es un buen indicador de estado nutricional ya que se correlacionó con el IMC en el grupo de estudio. En los sujetos estudiados la dinamometría de mano, tanto dominante como no dominante, se relacionó débilmente con el IMC, por lo que no sería un buen indicador del estado nutricional de adultos mayores de 60 años.

\section{RESUMEN}

Introducción: El control de la natalidad y el descenso de la mortalidad han desencadenado un envejecimiento demográfico, por lo que en algunos casos no es posible determinar con exactitud la estatura o el peso, careciendo de utilidad los indicadores nutricionales. Objetivo:

\section{FIGURA 2}

\section{Dinamometría de mano no dominante según estado nutricional de adultos mayores pertenecientes al Círculo de Carabineros $(\mathrm{R})$ de Chillán $(\mathrm{n}=60)$.}

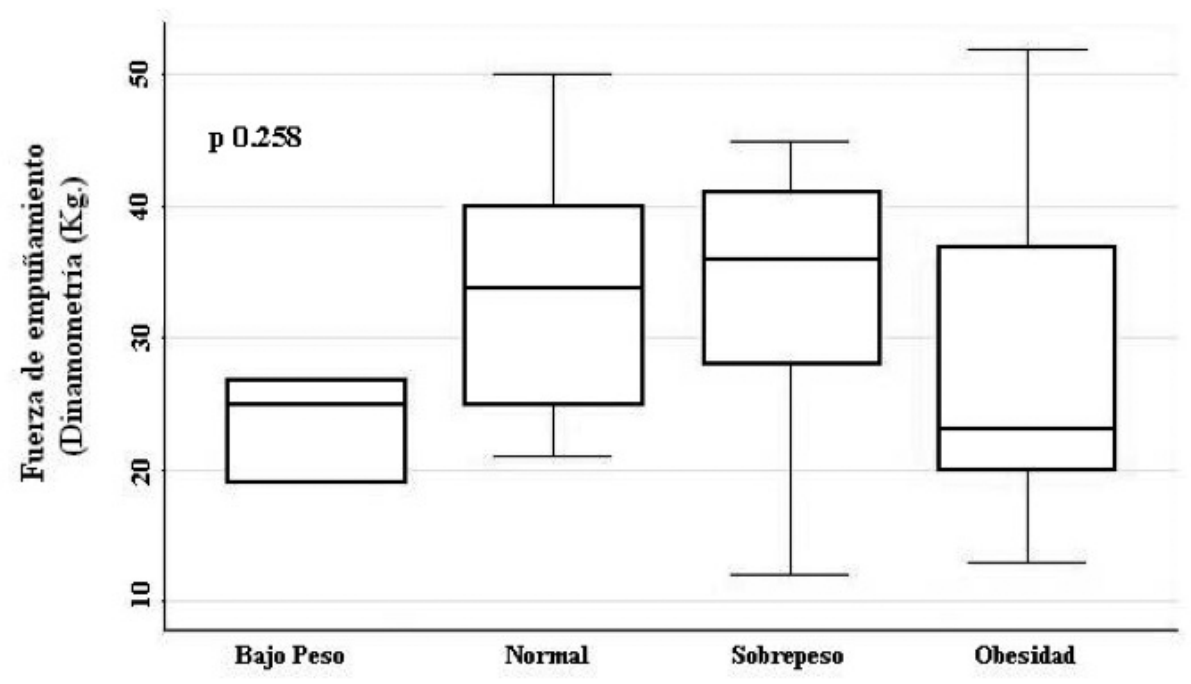


Relacionar el índice de masa corporal (IMC) con el perímetro de pantorrilla y la fuerza muscular medida por la dinamometría de mano dominante y no-dominante. Métodos: Se estudiaron 60 adultos mayores de 60 años, con capacidad funcional de autovalencia, los adultos pertenecían al círculo de Carabineros de Chile en retiro de la ciudad de Chillan en la VII Región. Los datos antropométricos y la dinamometría se determinaron de acuerdo a las técnicas establecidas por la OMS. Se utilizó el coeficiente de correlación y Anova para determinar la asociación entre las variables. Resultados: Se observó una relación negativa débil entre ambas dinamometrías con el IMC; $r=-0.19$, dominante ( $p 0.332$ ) y no dominante $\mathrm{r}=-0.11$ ( $\mathrm{p} 0.806$ ), pero se encontró una correlación positiva de carácter mediana, entre el perímetro de pantorrilla y el IMC, $r=0.64(p<0.001)$. Conclusiones: Los datos aportados evidencian la utilidad del perímetro de pantorrilla como indicador del estado nutricional en los sujetos de estudio, no así la dinamometría de mano.

Palabras clave: Estado nutricional, índice de masa corporal (IMC), fuerza muscular (dinamometría), perímetro de pantorrilla, ancianos.

Dirigir la correspondencia a:

Sr. José Luis Pino Villalón

Director Carrera de Nutrición y Dietética

Universidad del Mar, Sede Centro Sur

Campus Talca

Talca, Chile

4 Poniente 1223

Fono: (71) 417035 - 417000

Fax: 417062

E-mail:jose.pino@udelmar.c1

\section{BIBLIOGRAFIA}

1. Macionis John, Plummer Ken. Sociología, Madrid, España, Ediciones Prentice Hall, 1999.

2. Marin L Pedro Paulo. Tiempo nuevo para el adulto mayor; Capitulo 3; Segunda Edición; p 49. 1993.

3. Cabrera Hernández A, Jiménez Acosta S. Algunos factores de riesgo en enfermedades cardiovasculares en un grupo de adultos supuestamente sanos. Rev Cubana Aliment Nutr 1997;11(1):40-5.

4. López Masó IM, Rodríguez Hernández HM. Propuesta de intervención para ancianos deprimidos. Rev Cubana Med Gen Integr 1999;15(1):19-23.

5. Corman B, Teillet L, Micmel JB, Yu BP. Nutrition and the elderly. J Nutr 1997;1(3):133-41.

6. Alcaraz A. Maritza, Fong E. Juana A., Álvarez P. Norka y Pérez R. Arnoldo, Evaluación del estado nutricional del adulto mayor en el reparto Flores, MEDISAN 2001;5(1):46-51.
7. Chumlea W. y Sun S. Importancia de la composición corporal en el anciano J Nutr Health Aging. 2004; $8(2) ; 11$.

8. Ministerio de Salud, Departamento de Estadísticas e Información de Salud, población del adulto mayor en control, según estadode nutrición y sexo, Diciembre 2010 Chile www deis.minsal.cl

9. Urteaga R, Carmen; Ramos H, Rosa Isela, Atalah S, Eduardo. Validación del criterio de evaluación nutricional global del adulto mayor. Rev Méd Chil. 2001;129 (8), 871-6.

10. Hirsch S, De la Maza MP, Obaldía N, Espinoza J, Hübner C, Peterman M, Bunout D. Fureza Muscular: Un indicador de estado nutritivo. Rev Méd Chile 1992 ; 120: 615 - 20 .

11. W. Chumlea, G. Hall, et all; MNA y composición corporal en los adultos sanos; Mini Nutritional Assessment (MNA):investigación y práctica en las personas de edad avanzada; Nestlé Nutrition Workshop vol $1 ; 1998$ p. 11.

12. Bonnefoy M., Jauffret M., et all.; Usefulness of calf circumference measurement in assessing the nutritional state of hospitalized elderly people. Gerontol 2002;48:162-9.

13. Herrera Héctor, Rebato Esther, Rocandi Ana María et al. Caracterización antropométrica de una población de adultos mayores institucionalizados de la ciudad de Caracas, Venezuela. Invest Clin. 2005;46, (2)139-56.

14. Quinteros Dolly S. Técnicas para la toma de medidas antropométricas, Centro de atención Nutricional, Medellín; Segunda edición; p 11-32. 1992.

15. Jelliffe D B. Evaluación del estado nutricional de la comunidad. Ginebra: OMS; 1968:291 (Monografía 53).

16. Quetelet LAJ. Physique sociale, vol. 2. Brussels, C. Muquardt. p 92. 1869.

17. Aleman-Mateo Heliodoro; Perez Flavia A.;'Los indicadores del estado de nutrición y el proceso de envejecimiento". Nutr Clin 2003; 6 (1) 46-52.

18. Urtega R, Carmen; Ramos H, Rosa Isela y Atalah S, Eduardo. Validación del criterio de evaluación nutricional global del adulto mayor. Rev Méd Chil. 2001, 129 (8) 871-76.

19. Falque Madrid, Luis; Piñero Corredor, María; Zambrano de Rodríguez, Nelly; Quintero, Jorge; Souki de Gabarrón, Aida; Arias Márquez, Nelly. Estado nutricional y composición corporal de un grupo de adultos mayores no institucionalizados del Estado Zulia, Venezuela, Arch Latinoam Nutr. 1996; 46(3) 190-5.

20. Gisela P. Rodríguez, Consuelo M. Matos, y cols., 
Evaluación nutricional de un grupo de adultos mayores en un consultorio médico de familias, Rev Cubana Aliment Nutr 1999;13(2) 91-7.

21. Javier Aranceta-Bartrinaa, Lluís Serra-Majemb, Màrius Foz-Salac, Basilio Moreno-Esteband, y Grupo Colaborativo SEEDO, Prevalencia de obesidad en España. Med Clin (Barc). 2005;125(12) 460-6.

22. Varo J.J., Martínez-González M.A., Martínez J.A., Obesity prevalence in Europe, ANALES Sis San Navarra 2002; 25 (Supl. 1) 103-8.

23. World Health Organization, Global Database on Body Mass Index,[fecha de consulta 15/12/2010] disponible en: http://apps.who.int/bmi/index.jsp

24. Restrepo M, Sandra Lucia Et Al. Los hábitos alimentarios en el adulto mayor y su relación con los procesos protectores y deteriorantes en salud. Rev Chil Nutr 2006, 33 (3) 500-10.

25. Cuervo, M. et al. Valoración de la circunferencia de la pantorrilla como indicador de riesgo de desnutrición en personas mayores. Nutr Hosp 2009: 24 (1) p. 63-7.

26. Portero-McLellan KC, Staudt C, Silva FR, Delbue Bernardi JL, Baston Frenhani P, Leandro Mehri VA., The use of calf circumference measurement as an anthropometric tool to monitor nutritional status in elderly inpatients, J Nutr Health Aging. 2010:14(4) 266-70

27. Kubo A, Yoshimatsu T, Nishida Y., Relation of the maximum calf circumference with albumin and body mass index in elderly cases of chronic hospitalization, Nippon Ronen Igakkai Zasshi. 2002:46(3) 239-43

28. Wijhhovep HA, van Bokhorst-de van der Schweren MA Heymans MW, e Net HC, Kruizenga HM, Iwisk JW, Visser M, Low mid-upper arm circumference, calf circumference, and body mass index and mortality in older persons. J Gerontol A Biol Sci Med Sci 2010 Oct:65(10) 1107-14.

29. SolmanK,Stobäus N, Gonzalez MC, Schulzke JD, Pin lich M., Hand grip strength: Outcome predicfor and marker of nutritional status. Clin Nutr. 2010 Oct 20_Epwbahead of print]

30. Bohannon RW., Hand-grip dynampmetry predicts future outcomes in aging adults, J Geriatr Phys Ther. $2008 \cdot 31(1) 3-10$

31. Sch |iissel MM, dos Anjos LA, de Vasconcellos MT, Kac G., Reference values of handgrip dynamometry ff healthy adults: a population-based study. Clin Nutr 2008: 27(4) 601-7.

32. Bohannon RW. Dynamometer measurements of hand-grip strength predict multiple outcomes, Percept Mot Skills. 2001;93(2) 323-8.

33. Arroyo Patricia, Lera Lydia, Sánchez Hugo, Bunout Daniel, Santos José Luis, Albala Cecilia, Indicadores antropométricos, composición corporal y limitaciones funcionales en ancianos, Rev Méd Chi_ 2007: 135:846-54

34. Syddall H, Cooper C, Martin F, B riggs R, A ihlie Sayer A., Is grip strength a useful single marker of frailty? A ge Ageing 20 3;32(6) 650-6

35. Webb AR, Newman LA, Tay lor M, Keogh JB., Hand grip dynamometry as a predictor of postoperative complications reappraisal_using age standardjzed grip strengths. JPEN J Parenter Enteral Nutr. 1989;13(1) 30-3.

36. Rosales B, Valeria et al. Comparación entre nutrición enteral precoz y nutrición enteral Tardía En El Estado Nutricional De Pacientes Gastrectomizados Rev ChilN Nutr 2009.36 (1) 15-22

37. Arnold CM, Warkentin KD, Chillibeck PD, Magnus CR. The reliability and validity of handheld dynamometry for the measurement of lower-extremity muscle strength in older adults, J Strength Cond Res 2010:24(3)815-24.

38. Tsai AC, Ghang TL, Wang YC, Liap CY., Population-specific short-form mini nutritional assessment with body mass index or calf circumference can predict risk of malnutrition in community-living or institutionalized elderly people in taiwan, J Am Diet Assoc. 2010; 110(9)1328-34.

39. Tapia Jesús y cols., Parámetros objetivos regionales de evaluación nutricional en una población de adulto mayor, Nutr Clín 2003:6 (1) 27-35

40. Ali $N A, O$ B ien_IM_Ir, Hodfmann_SP, phillips $G$

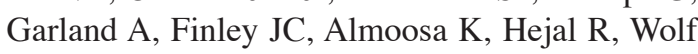
KM, Lemeshow S, Contnors AF Jr, Marsh CB, Acquired weakness, handgrip strength, and mortality incritically ill patients, Am I Respir Crit Care Med. 2008;178(3)261-8.

41. Emilio José García Mayo Participación del estado nutricional en la fragilización del individuo Artículo de revisión. Nutr Clín 2003;6(1)58-62.

42. Barbosa Murillo, J. A. P. et al. Masa muscular, fuerza muscular y otros componentes de funcionalidad en adultos mayores institucionalizados de la Gran Caracas-Venezuela. Nutr Hosp. 2007; 22 (5) 578-83.

43. Diaz Nayka, Meertens Lesbia, Solano Liseti et al. Caracterización nutricional antropométrica de ancianos institucionalizados y no institucionalizados. Invest Clin. 2005; 46 (2) 111-9. 